\title{
Comment
}

Neuro epidemiology

Neuroepidemiology 2013;41:116-117

DOI: $10.1159 / 000353212$

\section{The Timing of Tremor: A Biological Clock in Essential Tremor?}

\section{Julián Benito-León ${ }^{\mathrm{a}-\mathrm{c}}$}

aDepartment of Neurology, University Hospital '12 de Octubre', ${ }^{b}$ Department of Medicine, Faculty of Medicine, Complutense University, and ${ }^{\mathrm{C} C e n t r o}$ de Investigación Biomédica en Red sobre Enfermedades Neurodegenerativas (CIBERNED), Madrid, Spain

Essential tremor (ET) is one of the most common neurological diseases among adults, and it is the most common tremor disorder [1-7]. Head (neck) tremor is a major feature of ET, generally developing some time after the clinical expression of arm tremor. What drives the expression of this common clinical feature of ET? To date, investigators have not tried to unravel whether tremor duration, age, or both are driving the expression of this common yet inconstant feature of ET. If it were duration, this would suggest that it was a function of the natural evolution of the disease over time. If it were age, this would argue for the presence of a 'biological clock' that is driving clinical expression. The latter brings up the interesting notion that factors that are intrinsic to the patient could be interacting with their disease to modify its clinical expression.

In this issue of Neuroepidemiology, Louis [8] utilized cross-sectional data on more than $350 \mathrm{ET}$ patients of various ages and durations to untangle the effects of age from duration on the expression of head tremor in ET. Given the colinearity of age and duration, it is difficult to separate their effects without the use of statistical analyses. Each ET patient underwent a 20 -min videotaped neurological examination, which included an assessment of the presence/absence of head tremor. Head tremor was present on examination in $140(38.6 \%)$ patients. Of note was that young patients, including those with longer-duration tremor, rarely had head tremor. Thus, only $7.4 \%$ of the patients who were less than 40 years old (tremor duration $\geq 10$ years) had head tremor versus $42.8 \%$ of patients who were older than 60 (tremor duration $\geq 10$ years). The difference was highly significant. In multivariate models, while head tremor was associated with age $(\mathrm{p}<0.001)$, it was not independently associated with tremor duration $(\mathrm{p}=0.26)$. Indeed, with the exception of 1 patient, head tremor did not begin before age 36. In sum, these data, from a large sample of ET patients, indicate that the appearance of head tremor in ET is rare before the age of 40 and that its presence was determined more clearly by the patients' age rather than the duration of their tremor.

Another interesting finding of this study is that female gender seemed to predispose ET patients to develop head tremor. In Caucasians, head tremor is more common in women with ET than in men $[9,10]$. By contrast, in Taiwanese ET patients, this is not the case [11]. Therefore, other factors, such as race, could be interacting with their disease to modify its clinical expression.

The results of this study are of special interest, essentially because there are no prior studies that have unraveled the 'driver' of head tremor. These data are likely to be of some value in clinical settings, which are often enhanced by the availability of empirical knowledge and an enhanced understanding of clinical patterns.

The study had several strengths, including the large sample size, the extensive study of the patients and the well-executed statistical analyses. However, the study was not without limitations. Specifically, while age was known precisely, the precise age of onset of ET may be more difficult to establish. However, a recent study suggests that age of onset, overall, is reliably reported by ET patients $[12,13]$.

Leaving aside issues of strengths and limitations, the study demonstrates that the occurrence of head tremor in ET is a function of age rather than disease duration. In other words, the appearance of head tremor in ET seems to depend on a biological factor that is intrinsic to the patient (i.e., age or aging), and is not the independent consequence of advancing disease duration. With so little known about the biology of tremor, small clues, such as this, provide additional stepping stones for the next set of studies.

\section{Acknowledgements}

Dr. Benito-León is supported by R01 NS039422 from the National Institutes of Health, Bethesda, Md., USA and by ICT-2011287739 (NeuroTREMOR project) grant from the Commission of the European Union.

\section{KARGER}

E-Mail karger@karger.com

www.karger.com/ned
2013 S. Karger AG, Basel

0251-5350/13/0412-0116\$38.00/0
Dr. Julián Benito-León

Avda. de la Constitución 73

Portal $3,7^{\circ}$ Izquierda

ES-28821 Coslada (Spain)

E-Mail jbenitol@meditex.es 


\section{References}

$>1$ Benito-Leon J, Louis ED: Essential tremor: emerging views of a common disorder. Nat Clin Pract Neurol 2006;2:666-678;quiz 2p following 691.

$>2$ Benito-Leon J, Louis ED: Clinical update: diagnosis and treatment of essential tremor. Lancet 2007;369:1152-1154.

3 Benito-Leon J, Louis ED: Update on essential tremor. Minerva Med 2011;102:417-439.

4 Benito-Leon J: How common is essential tremor? Neuroepidemiology 2009;32:215-216.

5 Benito-Leon J: Essential tremor: one of the most common neurodegenerative diseases? Neuroepidemiology 2011;36:77-78.

6 Benito-Leon J, Bermejo-Pareja F, Morales JM, Vega S, Molina JA: Prevalence of essential tremor in three elderly populations of central Spain. Mov Disord 2003;18:389-394.

7 Benito-Leon J, Bermejo-Pareja F, Louis ED, Neurological Disorders in Central Spain Study Group: Incidence of essential tremor in three elderly populations of central Spain. Neurology 2005;64:1721-1725.
8 Louis ED: When do essential tremor patients develop head tremor? Influences of age and duration and evidence of a biological clock. Neuroepidemiology 2013;41:110-115.

$\checkmark 9$ Louis ED, Ford B, Frucht S: Factors associated with increased risk of head tremor in essential tremor: a community-based study in northern Manhattan. Mov Disord 2003;18:432-436.

10 Hubble JP, Busenbark KL, Pahwa R, Lyons K, Koller WC: Clinical expression of essential tremor: effects of gender and age. Mov Disord 1997; 12:969-972.

$\checkmark 11$ Chuang WL, Lu CS, Huang YZ, Chen RS: Clinical characteristics of essential tremor in Taiwan: an exploratory-comparative study. Eur J Neurol 2012;19:135-141.

12 Louis ED: Age of onset: can we rely on essential tremor patients to report this? Data from a prospective, longitudinal study. Neuroepidemiology 2013;40:93-98.

13 Benito-Leon J: 'When did my tremor start?': can essential tremor patients pinpoint their disease onset? Neuroepidemiology 2013;40:99-100. 